\title{
Instituições e nível de renda: uma abordagem empírica para os municípios paranaenses
}

\author{
Ana Elisa Gonçalves Pereira* \\ Luciano Nakabashi** $^{* *}$
}

\begin{abstract}
RESUMO - O estado do Paraná apresenta uma grande disparidade no nível do PIB por trabalhador em seus municípios. A diferença entre os municípios com o maior e o menor PIB por trabalhador no início desta década, segundo dados do IBGE (2000), era superior a $700 \%$. Estudos para tentar detectar as causas dessa má distribuição são de grande relevância. Uma explicação para as disparidades nos níveis de renda advém da teoria institucionalista. Diversos estudos empíricos nesse campo encontram uma elevada correlação entre o grau de desenvolvimento das instituições e a renda per capita. A teoria sugere que as instituições afetam a renda e seu crescimento por meio da distribuição de poder político, da geração de oportunidades econômicas, do estímulo à inovação e à acumulação de capital humano, além de outras vias. Tendo isso em vista, o objetivo do presente artigo consiste em mensurar a qualidade das instituições municipais paranaenses e avaliar o efeito que elas exercem sobre o produto por trabalhador dos municípios, tomando como base o arcabouço teórico institucionalista.
\end{abstract}

Palavras-chave: Instituições. Crescimento econômico. Economia paranaense.

\section{INTRODUÇÃO}

O estado do Paraná apresenta uma grande disparidade no nível do PIB por trabalhador em seus municípios. A diferença entre os municípios com o maior e o menor PIB por trabalhador no início desta década, segundo dados do IBGE (2000), era superior a 700\%. Estudos para tentar detectar as causas dessa má distribuição são de grande relevância.

Uma explicação para as disparidades nos níveis de renda advém da teoria institucionalista. Diversos estudos empíricos nesse campo encontram uma elevada correlação entre o grau de desenvolvimento das instituições e a renda per capita. A teoria sugere que as instituições afetam a renda e seu crescimento por meio da distribuição de poder político, da geração de oportunidades econômicas, do estímulo à inovação e à acumulação de capital humano, além de outras vias.

\footnotetext{
* Graduanda em Economia pela Universidade Federal do Paraná. Endereço eletrônico: anaelisagpereira@gmail.com.

** Doutor em Economia pelo CEDEPLAR/UFMG. Professor do Departamento de Economia da Universidade Federal do Paraná. Endereço eletrônico: luciano.nakabashi@ufpr.br.
} 
Tendo isso em vista, o objetivo do presente artigo consiste em mensurar a qualidade das instituições municipais paranaenses e avaliar o efeito que elas exercem sobre o produto por trabalhador dos municípios, tomando como base o arcabouço teórico institucionalista.

\section{DESENVOLVIMENTO E INSTITUIÇÕES NO PARANÁ}

No Brasil, apesar da grande dimensão territorial, há uma uniformidade das macroinstituições - democracia, presidencialismo, idioma, sistema judiciário, entre outras que geralmente são utilizadas como proxies para a variação da qualidade institucional nas análises entre países. No entanto, não se pode afirmar que a qualidade das instituições políticas e econômicas, ao menos em sua dimensão de facto, é homogênea ao longo do território nacional e mesmo dentro de uma região do país. Existem diferenças nessas instituições, tanto entre estados quanto entre municípios.

Pode-se constatar, adicionalmente, a elevada disparidade nos níveis de renda, acumulação de capital físico e humano entre estados, regiões e municípios. Entender a origem de tais disparidades - visando, em última instância, obter meios de reduzi-las - consiste numa tarefa complexa, que está sempre entre os principais objetivos de pesquisadores e formuladores de política econômica.

No estado do Paraná, observa-se grande disparidade nos níveis de renda. Mesmo no início do atual milênio, a diferença entre o menor e o maior PIB por trabalhador - referentes aos municípios de Tijucas do Sul e São José dos Pinhais, respectivamente - superava os 700\% (IBGE, 2000).

Por meio da análise de indicadores de qualidade das instituições municipais, pode-se analisar se essa variável é capaz de explicar parte significativa de tais disparidades econômicas.

\subsection{DADOS MUNICIPAIS}

No presente estudo, utilizamos como objeto de análise uma amostra contendo 290 municípios do estado do Paraná - dentre os 403 considerados pelo IBGE - para os quais há dados disponíveis. Muitos dados considerados em análises interestaduais e entre países não estão disponibilizados de forma desagregada por município. Também foram excluídos os outliers, pois estes poderiam distorcer os resultados da análise. Desse modo, a amostra reduziuse a 285 observações. 
2.1.1 Variável a ser explicada e controles

O objetivo do presente estudo é analisar a relação entre as instituições e o Produto Interno Bruto (PIB) por trabalhador dos municípios, que consiste na razão PIB/população acima de 15 anos. Os dados foram obtidos do Censo 2000 do Instituto Brasileiro de Geografia e Estatística (IBGE).

Adicionalmente, utilizamos como variáveis de controle outras duas medidas, que refletem aspectos referentes à acumulação de capital físico e humano nos municípios. São elas, respectivamente, o consumo médio de energia por trabalhador e a média de anos de estudo da população acima de 25 anos. Ambas as medidas foram obtidas do mesmo Censo 2000 do IBGE.

\subsubsection{Medidas de qualidade das instituições locais}

Nesta análise empírica, utilizamos dois indicadores da qualidade institucional municipal, definidos como INST e IQIM. O primeiro índice foi construído - com base no estudo de Naritomi (2007) - para avaliar três dimensões do arcabouço institucional dos municípios. O segundo é o Índice de Qualidade Institucional Municipal, disponibilizado pela Agenda Político-Institucional do Ministério do Planejamento, Orçamento e Gestão.

O primeiro índice de qualidade institucional dos municípios - nomeado INST consiste na união de três subindicadores: índice de Gini da distribuição de terras; índice de concentração política; e índice de acesso à justiça.

O índice de Gini da distribuição de terras foi calculado para cada município com base nos dados do Censo Agrícola 1996 (IBGE), utilizando-se a proporção acumulada do número de estabelecimentos e a proporção acumulada da área, por estrato de área total.

Quanto à concentração política, utilizaram-se os dados da eleição de 2000 para vereador, obtidos do Tribunal Superior Eleitoral. Calculou-se o índice de HirschmanHerfindahl da soma dos quadrados das parcelas dos votos obtidos por cada partido político. Supõe-se, com base na literatura nacional e internacional, que quanto maior a concentração política, menos favoráveis seriam as instituições vigentes ao desenvolvimento econômico. 
Já o índice de acesso à justiça consiste na soma simples de três variáveis binárias indicando a existência ou não (nos municípios em 2000) de: i) Tribunal de Pequenas Causas ${ }^{1}$; ii) Conselho Tutelar; e iii) Comissão de Defesa do Consumidor. (Apêndice 3).

Visto que o sinal do impacto desses três subindicadores sobre a qualidade institucional não é o mesmo - acesso à Justiça influi positivamente, enquanto os demais influem negativamente - o primeiro indicador de qualidade institucional INST resultou da soma Acesso à Justiça $+(1$ - Concentração Política $)+(1$ - Gini da Terra $)$, cada indicador variando de 0 a 1 . Dessa forma, obteve-se um indicador que assume valores de 0 a 3 , no qual quanto mais próximo de 3, melhor a qualidade das instituições (no sentido de promoção do crescimento).

O segundo indicador das instituições dos municípios utilizado na análise empírica é o Indicador de Qualidade Institucional Municipal (IQIM), elaborado pelo Ministério do Planejamento para os 5.507 municípios brasileiros existentes no período 1997-2000 (última base disponibilizada pelo IBGE).

O indicador resulta da soma com pesos iguais de três conjuntos de subindicadores: i) o Grau de Participação - que procura mensurar a participação da população na administração municipal, partindo do número de Conselhos Municipais e suas características; ii) a Capacidade Financeira - que afere o número de consórcios intermunicipais, a relação entre a dívida do município e as suas receitas correntes, líquidas das despesas de pessoal (o que reflete sua capacidade de quitar essa dívida no tempo), e a Poupança Real per capita; e iii) a Capacidade Gerencial - que indica a atualidade da planta de valores para fins de IPTU, o grau de adimplência em relação ao mesmo tributo e o número de instrumentos de gestão ${ }^{2}$ e planejamento utilizados pelo poder municipal.

\subsubsection{Variáveis geográficas}

Ambos os indicadores da qualidade institucional foram utilizados para mensurar a correlação entre o arcabouço institucional municipal e o crescimento econômico. A análise da causalidade, porém, quando realizada pelo método dos Mínimos Quadrados Ordinários

\footnotetext{
1 Apesar de o IPEADATA utilizar a denominação Tribunal de Pequenas Causas, tal órgão tem sido designado Juizado Especial desde a lei 9099 de 1995.

2 Os instrumentos de gestão seriam: existência de Administração Distrital ou Regiões Administrativas, Subprefeitura, Plano Diretor, Lei de Parcelamento do Solo, Lei de Zoneamento ou equivalente, Código de Obras e Código de Posturas; e os instrumentos de planejamento: existência de Plano de Governo, Plano Estratégico e Lei Orgânica.
} 
(MQO), pode fornecer estimadores tendenciosos e inconsistentes, superestimando o impacto das instituições devido à questão da endogeneidade, pois os municípios com renda mais elevada podem ser capazes de desenvolver um arcabouço institucional melhor. Deve-se, portanto, buscar instrumentos exógenos para representar a variação institucional e estimar a influência das instituições sobre o desenvolvimento econômico.

Usualmente, aspectos geográficos como a latitude, as temperaturas, as chuvas e a distância da costa são utilizados como variáveis instrumentais para a qualidade das instituições (HALL; JONES, 1998, ENGERMANN; SOKOLOFF, 2002, EASTERLY; LEVINE, 2002, MENEZES-FILHO et al., 2006). No Brasil, por exemplo, nota-se um claro padrão geográfico quando se compara desenvolvimento das instituições, renda per capita, mortalidade infantil e anos de estudo em função da latitude: estados e municípios mais próximos do equador apresentam, em geral, desenvolvimento inferior aos mais distantes. Porém, para o caso dos municípios paranaenses, a latitude não parece tão relevante, dada sua pequena variação.

Neste trabalho, opta-se pela instrumentação das instituições por meio de variáveis geográficas, certamente exógenas em relação à renda corrente dos municípios. A variável geográfica que apresentou correlação mais significativa com a qualidade institucional - tanto medida pelo indicador construído da qualidade institucional (INST) quanto pelo IQIM - é a média anual de temperaturas. A correlação negativa entre as variáveis denota que quanto maiores as temperaturas no município, menores são os índices de qualidade das instituições, ou seja, menos favoráveis são as instituições ao desenvolvimento econômico.

Uma possível explicação para essa correlação negativa é a influência desse fator geográfico nas fases iniciais de conformação das instituições econômicas dos municípios. Nas regiões de clima mais quente, as atividades agrícolas baseadas em grandes propriedades se desenvolveram de forma mais expressiva, propiciando o desenvolvimento de instituições menos favoráveis à geração de oportunidades de uma maneira mais ampla e, desse modo, gerando impactos negativos na industrialização e no crescimento econômico desses municípios.

A qualidade institucional foi, portanto, instrumentada pela média de temperaturas, no primeiro estágio das regressões de Mínimos Quadrados em Dois Estágios (MQ2E) que buscaram mensurar o impacto da qualidade institucional sobre o PIB por trabalhador. 


\section{EVIDÊNCIA EMPÍRICA}

\subsection{ESPECIFICAÇÃO}

A estratégia para não incorrer no problema da endogeneidade das instituições é a utilização de variáveis instrumentais exógenas, pelo método de Mínimos Quadrados em Dois Estágio (MQ2E). No primeiro estágio, tem-se que:

$$
W_{i}=\alpha_{0}+\alpha_{1} Z_{i}+\alpha_{2} K_{i}+\alpha_{3} H_{i}+\varepsilon_{i}^{\prime}
$$

onde: $W_{i}$ é o vetor de qualidade institucional, $Z_{i}$ é a média anual de temperaturas, $K_{i}$ é o consumo de energia elétrica não-residencial por trabalhador, $H_{i}$ é a média de anos de estudo e $\varepsilon_{i}^{\prime}$ é o termo de erro.

Por meio da equação (2) pode-se estimar os valores de $W_{i}$, denominados $\hat{W}_{i}$. No segundo estágio, utiliza-se $\hat{W}_{i}$ - instrumentado por $Z_{i}$ e as variáveis de controle $K_{i}$ e $H_{i}-$ como regressor, ao invés da variável endógena $W_{i}$.

$$
Y_{i}=\beta_{0}^{\prime}+\beta_{1}^{\prime} \hat{W}_{i}+\beta_{2}^{\prime} K_{i}+\beta_{3}^{\prime} H_{i}+\varepsilon_{i}^{\prime \prime}
$$

A equação (3) mensura o efeito da qualidade das instituições, instrumentada pela variável geográfica em questão, sobre o PIB por trabalhador, controlando para as proxies para capital físico e humano em cada município.

\subsection{RESULTADOS}

Nas tabelas que seguem, são apresentados os resultados empíricos dos estágios descritos na subseção anterior. A tabela 1 apresenta os resultados da estimação das regressões pelo método de MQ2E.

A variável $K$ mede o consumo de energia por trabalhador e $H$, a escolaridade média. A variável explicada $Y$ é o PIB por trabalhador. As colunas (1) e (2) apresentam os resultados do $1^{\circ}$ e $2^{\circ}$ estágios, respectivamente, da estimação por MQ2E da influência do Índice de Qualidade Institucional Municipal (IQIM) - representado por W1 - sobre o PIB por trabalhador $(Y)$. Na regressão (1), o IQIM é instrumentado pela média anual de 
temperaturas $(Z)$. Na regressão (2), os valores estimados de $W 1$ são incluídos como regressores.

Os resultados sugerem que as temperaturas relacionam-se negativamente com a qualidade das instituições ( $Z$ é significativo a $1 \%$ ). A proxy para capital físico, $K$, não parece significativa para explicar o arcabouço institucional, porém o capital humano, aqui medido em $H$, apresenta um efeito positivo significativo a $1 \%$. Cada ano a mais na escolaridade média do município, de acordo com (3), corresponderia a uma diferença de aproximadamente 0.19 no indicador de qualidade institucional IQIM.

TABELA 1 - IMPACTO DAS INSTITUIÇÕES SOBRE A RENDA - VARIÁVEIS INSTRUMENTAIS

\begin{tabular}{|c|c|c|c|c|}
\hline & $\begin{array}{c}(1) \\
1^{\circ} \text { Estágio } \\
\end{array}$ & $\begin{array}{l}\text { MQ2E } \\
\text { (2) } \\
2^{\circ} \text { Estágio } \\
\end{array}$ & $\begin{array}{c}(3) \\
1^{\circ} \text { Estágio } \\
\end{array}$ & $\begin{array}{c}\text { (4) } \\
2^{\circ} \text { Estágio }\end{array}$ \\
\hline & $W 1$ & $Y$ & $W 2$ & $Y$ \\
\hline$W 1$ & & $\begin{array}{r}3.751106 \\
(1.97)^{* *}\end{array}$ & & \\
\hline$W 2$ & & & & $\begin{array}{l}9.161731 \\
(2.83) * * *\end{array}$ \\
\hline$K$ & $\begin{array}{r}0.0211743 \\
(0.87)\end{array}$ & $\begin{array}{r}0.7126952 \\
(4.45)^{* * *}\end{array}$ & $\begin{array}{r}-0.0052358 \\
(-0.95)\end{array}$ & $\begin{array}{l}0.497772 \\
(6.52) * * *\end{array}$ \\
\hline$H$ & $\begin{array}{r}0.1882627 \\
(5.71)^{* * *}\end{array}$ & $\begin{array}{r}0.4501567 \\
(1.17)\end{array}$ & $\begin{array}{r}0.1626365 \\
(7.47)^{* * *}\end{array}$ & $\begin{array}{r}0.2548127 \\
(0.661)\end{array}$ \\
\hline$Z$ & $\begin{array}{r}-0.0416479 \\
(-3.21)^{* * *}\end{array}$ & & $\begin{array}{r}-0.0382853 \\
(-4.38)^{* * *}\end{array}$ & \\
\hline$\alpha_{0}, \beta_{0}^{\prime}$ & $\begin{array}{r}3.243759 \\
(11.35)^{* * *}\end{array}$ & $\begin{array}{r}-8.204349 \\
(0.087)^{*}\end{array}$ & $\begin{array}{l}1.760646 \\
(9.05)^{* * *}\end{array}$ & $\begin{array}{l}-10.33945 \\
(-2.84) * * *\end{array}$ \\
\hline $\mathrm{R}^{2}$ & 0.144 & 0.2419 & 0.1963 & 0.2416 \\
\hline $\mathrm{R}^{2 *}$ & 0.1347 & 0.2337 & 0.1879 & 0.2336 \\
\hline
\end{tabular}

FONTE: elaboração própria.

NOTAS: Os testes t estão entre parênteses.*Significativo ao nível de $10 \%$, **Significativo ao nível de $5 \%$; ***Significativo ao nível de $1 \%$. $\mathrm{R}^{2 *}$ é o $\mathrm{R}^{2}$ ajustado. As variáveis explicadas são: (3) $W 1$, (4) $Y$, (5) W2, (6) $Y$, sendo apresentadas na quarta linha. As regressões de primeiro estágio seguem a especificação da equação (1) da subseção anterior, enquanto as regressões de segundo estágio seguem a especificação da equação (2). A variável $W$ é representada por $W 1$ nas duas primeiras colunas e por $W 2$ nas duas últimas.

No segundo estágio, observa-se que a qualidade das instituições, agora instrumentada pelas temperaturas, impacta positivamente sobre o PIB por trabalhador ( $\hat{W} 1$ é significativo a $5 \%$ ). O coeficiente de $\hat{W} 1$ sugere que a diferença de 1 ponto no indicador IQIM traduz-se em uma variação de $\mathrm{R} \$ 3.750,00$ no PIB por trabalhador. 
Embora fosse esperado que o estimador $\beta_{1}$ estivesse superestimando o impacto das instituições sobre o PIB, o método MQ2E aponta para uma influência superior à estimada $\operatorname{por} \operatorname{MQO}\left(\beta_{1}^{\prime}>\beta_{1}\right)$.

Um resultado interessante é a significância dos controles capital físico e humano no segundo estágio. A proxy para capital físico $K$ - que não se mostrou significativa no primeiro estágio para explicar a qualidade institucional - é significativa na determinação do PIB. Isso significa que investimentos em capital físico são relevantes na determinação do PIB por trabalhador de forma direta.

Já a proxy para capital humano $H$ deixa de ser significativa no segundo estágio. Pode-se inferir que a educação apresenta um efeito indireto sobre a renda, via instituições, porém não afeta diretamente o PIB quando se controla para a qualidade institucional. De acordo com os argumentos teóricos apresentados anteriormente, o impacto do capital humano sobre as instituições se deve, pelo menos em parte, a uma melhor distribuição da renda conforme ocorre um avanço no nível de escolaridade dos diferentes municípios. A melhora na distribuição de renda, por sua vez, provoca uma mudança no poder político de facto com efeitos positivos sobre o desenvolvimento das instituições de cada município paranaense.

As colunas (3) e (4) apresentam os resultados do $1^{\circ}$ e $2^{\circ}$ estágio, respectivamente, da estimação por MQ2E da influência do segundo indicador de qualidade das instituições, INST - representado por $W 2$-, sobre o PIB por trabalhador $(Y)$. Na regressão (3), o IQIM é instrumentado pela média anual de temperaturas $(Z)$. Na regressão (4), os valores estimados de $W 2$ são incluídos como regressores.

Os resultados obtidos, tanto no primeiro quanto no segundo estágio, são semelhantes. Além de denotar um efeito positivo da qualidade institucional sobre o produto por trabalhador, as regressões sugerem o efeito indireto da escolaridade sobre o PIB - por meio de seu impacto no arcabouço institucional - e negam o efeito direto da educação sobre o PIB.

\section{CONSIDERAÇÕES FINAIS}

A análise empírica demonstrou que ambos os indicadores de qualidade das instituições empregados são significativos para explicar diferenças no PIB por trabalhador entre os municípios do estado. Para contornar o problema da possível causalidade reversa 
entre renda e instituições, utilizou-se o método de Mínimos Quadrados em dois estágios, empregando como instrumento a média de temperaturas - variável evidentemente exógena e correlacionada com a qualidade institucional municipal.

Verificou-se que, controlando para a escolaridade média e o consumo médio de energia por trabalhador (proxies para estoque de capital humano e físico, respectivamente), diferenças nas instituições podem explicar diferenças expressivas no nível de produto por trabalhador nos municípios paranaenses.

Adicionalmente, os resultados apontaram para a existência de um efeito indireto da escolaridade sobre o desempenho econômico - por meio de seu impacto na qualidade institucional - e negaram o efeito direto da educação sobre o PIB, quando se inclui a qualidade institucional como controle.

Embora a hipótese de inércia institucional aponte para a permanência das instituições por um longo período de tempo, a teoria institucionalista admite possíveis reversões do quadro institucional. $O$ estudo da inter-relação entre instituições e desenvolvimento pode ser, portanto, um campo fértil de pesquisa rumo a um crescimento mais acelerado. Promovendo melhoras no arcabouço institucional dos municípios e regiões, formuladores de política econômica estarão primando pelo desenvolvimento econômico, reduzindo as expressivas desigualdades regionais que se observa no estado do Paraná.

\section{REFERÊNCIAS}

EASTERLY, W.; LEVINE, R.. Tropics, Germs, and Crops: how endowments influence economic development. National Bureau of Economic Research, Cambridge, 2002. Disponível em: < http://www.nber.org/papers/w9106>. Acesso em: 14/05/2008.

ENGERMAN, S. L.; SOKOLOFF, K. L. Factor endowments, inequality and paths of development among new world economics. National Bureau of Economic Research, Cambridge, 2002. Disponível em: < http://www.nber.org/papers/w9259>. Acesso em: 02/08/2008.

HALL, R. E.; JONES, C. I. Why some countries produce so much more output per worker than others? National Bureau of Economic Research, Cambridge,1998. Disponível em: < http://www.nber.org/papers/w6564>. Acesso em: 09/08/2008.

IBGE - Perfil dos Municípios Brasileiros - Pesquisa de Informações Básicas

Municipais 1999, 2001. IBGE, Rio de Janeiro.

IPEADATA - disponível em <www.ipeadata.gov.br>

MENEZES-FILHO, N.; MARCONDES, R.L.; PAZELLO, E.T.; SCORZAFAVE, L.G. Instituições e Diferenças de Renda entre os Estados Brasileiros: Uma Análise Histórica. In: XXXIV ENCONTRO NACIONAL DE ECONOMIA, 2006, Salvador. Anais do XXXIV Encontro Nacional de Economia, Salvador, 2006. CD-ROM. 
Ministério do PlAnEjAMENTO, ORÇAMENTO E GESTÃo. Agenda PolíticoInstitucional. Disponível em:

<http://www.planejamento.gov.br/secretarias/upload/Arquivos/spi/downloads/081014_D

OWN_EX_PC_Agen_sumAgenda.pdf $>$ Acesso em:

NARITOMI, J. Herança Colonial, Instituições e Desenvolvimento. Dissertação (mestrado). Programa de Pós-Graduação em Economia, Pontifícia Universidade Católica do Rio de Janeiro, Rio de Janeiro, 2007. 